\title{
Conversion Technology of Clothing Patterns from 3D Modelling to 2D Templates Based on Individual Point-Cloud
}

\author{
Tingyu XU, Huanyun WEI, Yue XIN, Longlin ZHANG* \\ College of Textile \& Garment, Southwest University, Chongqing, China China \\ DOI: $10.15221 / 15.134$ http://dx.doi.org/10.15221/15.134
}

\begin{abstract}
The research and application of clothing 3D technology focus on the 3D scanning data collection and analysis, the physical modelling based on the historical records, and the fitting and showing of virtual dynamics in China at present. In our research work of Three-dimensional Digital Studio, we will conduct a set of 3D scanning experiments between a body and a coating of the same object. Then we will make up a research chain of clothing patterns from $3 \mathrm{D}$ modelling to $2 \mathrm{D}$ templates based on individual, combining the usage of reverse engineering software to extract curves and surfaces of key parts. As an important result, we are going to offer an innovative research method to solve the study on the parameters relationship of space between human body and clothing, which will also offer a practical solution to the problems of 3D design and pattern flattening for loose clothing.
\end{abstract}

Key words: 3D scanning, individual Point-cloud, reverse engineering, 3D modeling, loose clothing

From the perspective of clothing structure, the ways of accessing template haven't been changed in a long time, including the design process of traditional clothing style and the making process of the pattern. The application of non-contact body-scanning technology in garment industry, which has made the virtual garment design become possible. The overall research plan of this topic is to collect data by non-contact body scanning technology, and set up standard 3D human body model, which is the basis of basic clothing pattern to solve the problem of patterns from 3D Modelling to $2 \mathrm{D}$ templates.

\section{Workflow}

In this experiment, we used Beijing CaMega 3D optical scanner from Boweihengxin, and the grating method was adopted non-contact body scanning to set up human body database. With a female body of size 160/84A, we referred it as intermediate-body. The first step is to obtain Point-cloud data by scanning. Then using the matching data acquisition and data processing software of plane curve extraction, the standard model of female intermediate-body was generated. At last, with a case study of shirt which can adjust the size and style, 2D templates were developed eventually ${ }^{[1]}$ (Fig. 1).

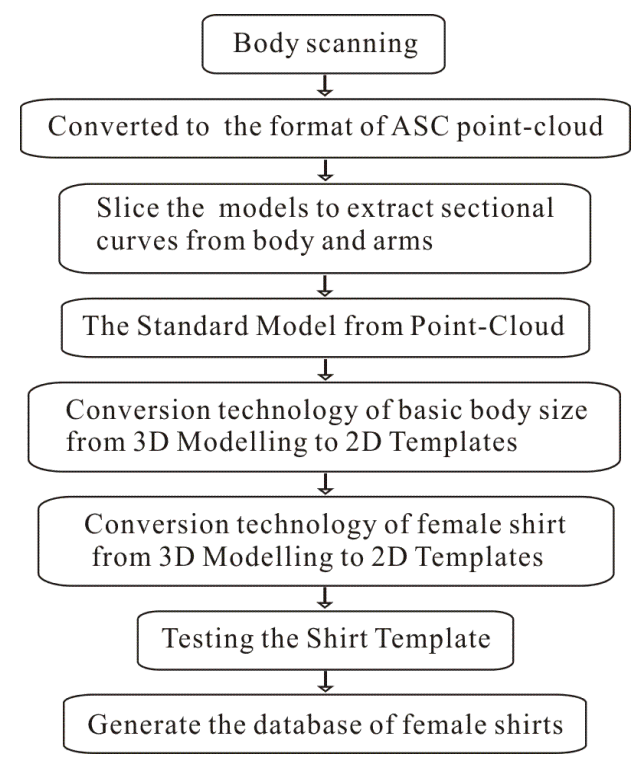

Fig. 1. Workflow for resizable shirt template.

* myfashionworks@163.com 


\section{The standard modelling of intermediate-body from Point-cloud data}

According to the second order stratified cluster sampling method, we selected five women size close to $160 / 84$ as samples to measure and convert to ASC point-cloud format. Then we got five groups of effective point-cloud image (figure 2). Using Geomagic Studios software to extract cross section data, extraction, sorting and analysis of data were done completely at the same time.
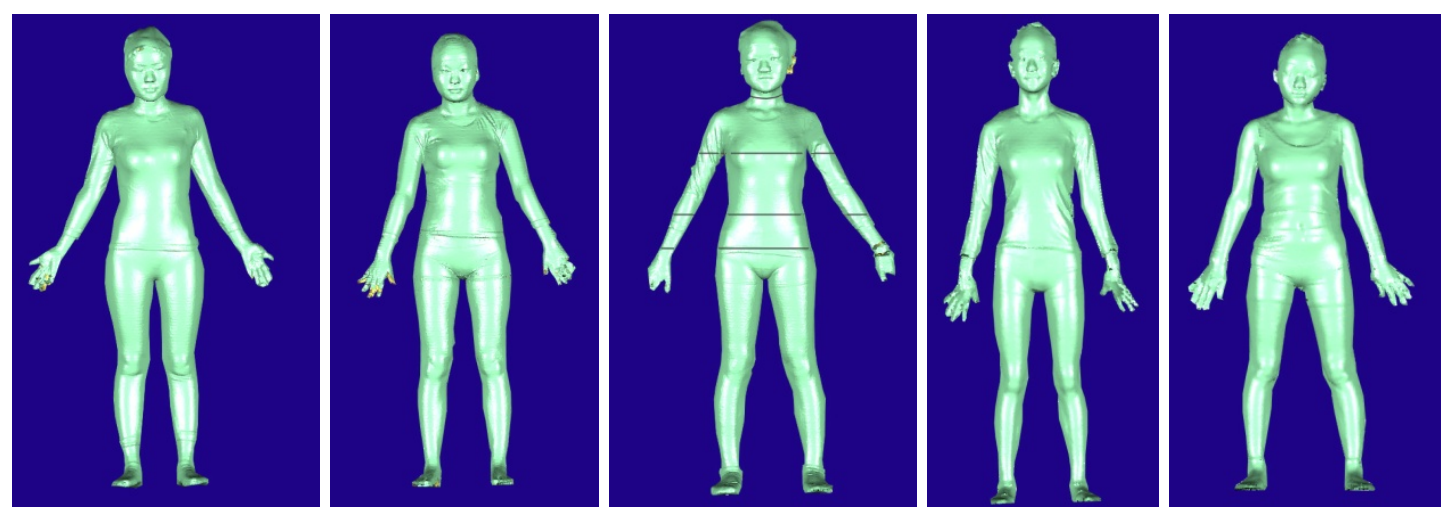

Fig. 2. Body measurements of the scanning subject.

There were 18 key parts measurement of 3D model, including head (surrounded degree, height), neck (surrounded degree, height), shoulder (surrounded degree, thickness, slope, height), back (width, thickness), height, body length, body length, arm length, chest, waist, abdomen, buttocks, thigh, knee, foot, arm, elbow, wrist, surrounded degree, thickness and width. Then every human body data and its height form graphic center to ground position were weighted calculation. Firstly, principal component analysis was used to find the first principal component and the second one. Secondly, with the first principal component of height weight, we carried on the regression weight of the remaining parts. Finally, we got the weighted average of the standard model data. The key data needed is as the following Table $1^{[2]}$.

Table 1. Body measurements of the scanning subject.

\begin{tabular}{lccccccc}
\hline & & \multicolumn{5}{c}{ Measurement in cm } \\
\hline & & \multicolumn{7}{c}{ Feasurement Positions } & Famale 1 & Famale 2 & Famale 3 & Famale 4 & Famale 5 & Middle Body \\
\cline { 2 - 7 } 1 & Height & 161 & 160 & 160 & 159 & 160 & 160.0025 \\
2 & Neck girth & 33.7 & 32.8 & 33.6 & 34.1 & 33.5 & 33.5395 \\
3 & Shoulder breadth & 38.5 & 38.2 & 38.6 & 39.2 & 38.4 & 38.5791 \\
4 & Chest girth & 83 & 85 & 84 & 86 & 82 & 83.9963 \\
5 & Waist girth & 67.8 & 68 & 69.4 & 68.5 & 68.2 & 68.3791 \\
6 & Hip girth & 88 & 92 & 87 & 90 & 88 & 88.9975 \\
7 & Upper arm girth & 24.6 & 24.2 & 22.8 & 26.6 & 24.0 & 24.4375 \\
8 & Arm length & 50.8 & 50.2 & 50.5 & 49.5 & 51.0 & 50.4016 \\
9 & Elbow girth & 20.2 & 20.6 & 20.5 & 21 & 20 & 20.4590 \\
10 & Wrist girth & 14.5 & 14.1 & 15.2 & 15.5 & 14.6 & 14.7788 \\
\hline
\end{tabular}


According to the key measurement of intermediate-body's standard model, we can get the data coil. Then input these coils into 3DMax software, and adopt the method of curved surface modeling to adjust and rearrange. According to wiring control grid, connect all close control grid, grid, cross section lines to get complete human body complement, and form female standard model (Fig. 3).

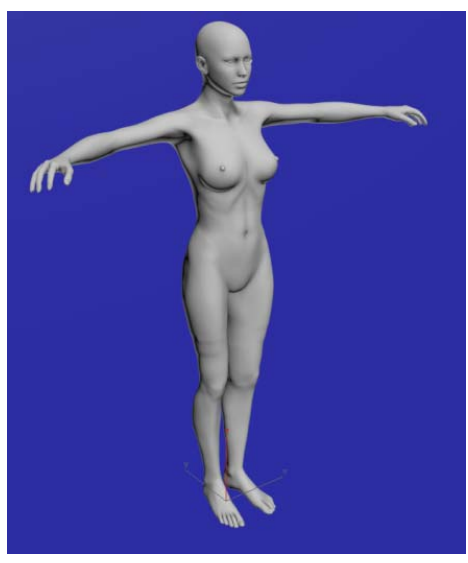

Fig. 3. The standard model of female middle body.

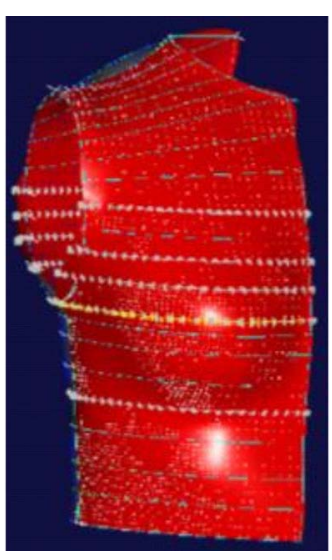

Fig. 4. Extracted date.

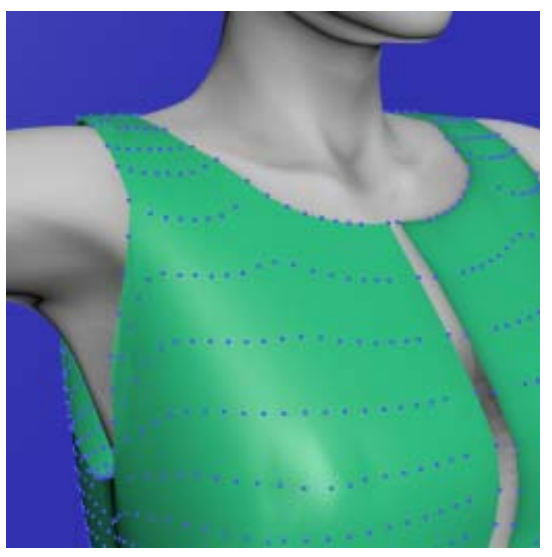

Fig. 5. The correspondence between body and model.

\section{Basic body templates from 3D to 2D}

\subsection{The data extraction of basic body model}

The experimental study object was the female's upper body pattern, so we picked the upper pointcloud data from the systemic point-cloud interception. Because clothing plate making are generally symmetry operation, we need only to reconstruct the right half point-cloud of 3D model (Fig. 4).

For a net body, use observation method to arrange along the navel from intercept point perpendicular to the horizon. Or adjusting the view to look down and showing the grid, the point-cloud will be subjected to a certain line around symmetrically placed after the interception. After the interception, the point-cloud with left and right side to render display, check point-cloud external features, and choose relatively smooth with obvious characteristics' right side point-cloud as the basis of point-cloud model.

After the construction of the model of point-cloud, extract the curve of each layer from reverse software and reconstruct 3D surface model. Curve the point-cloud according to the prototype pattern needed to extract the corresponding to each point-cloud, then each point-cloud in reverse software to synthesis of the corresponding curve, and set aside clothes chest, waist, hip as basis quantity. According to the drawing prototype pattern production lines of shoulder, chest circumference, waist line, side seam, chest wide line, back line, etc., these curves need to adjust control points to achieve the requirement of the final surface. Build a surface set into the female intermediates standard model of counterpoint, and amend the inappropriate parts ${ }^{[3]}$ (Fig. 5).

\subsection{Conversion technology of clothing patterns from 3D modelling to $2 \mathrm{D}$ templates}

In the process of transformation from 3D modelling to 2D templates, because of front panel chest bump part, the D-value of chest and waist will produce certain margin. In order to make the dress to fit the body, need to set up dart and rules. And in the position of the shoulder blade to open a small shoulder province or collar, make clothing more in line with human body characteristic.

As soon as surface integral operation is completed, the next is the process in DCTT (Design Concept Tech Tex). DCTT is France Lectra especially designed for the industrial fabrics market. Using DCTT border on the 3D model drawing template to automatically expand it to 2D templates (Fig. 6 ), used in the sample. 

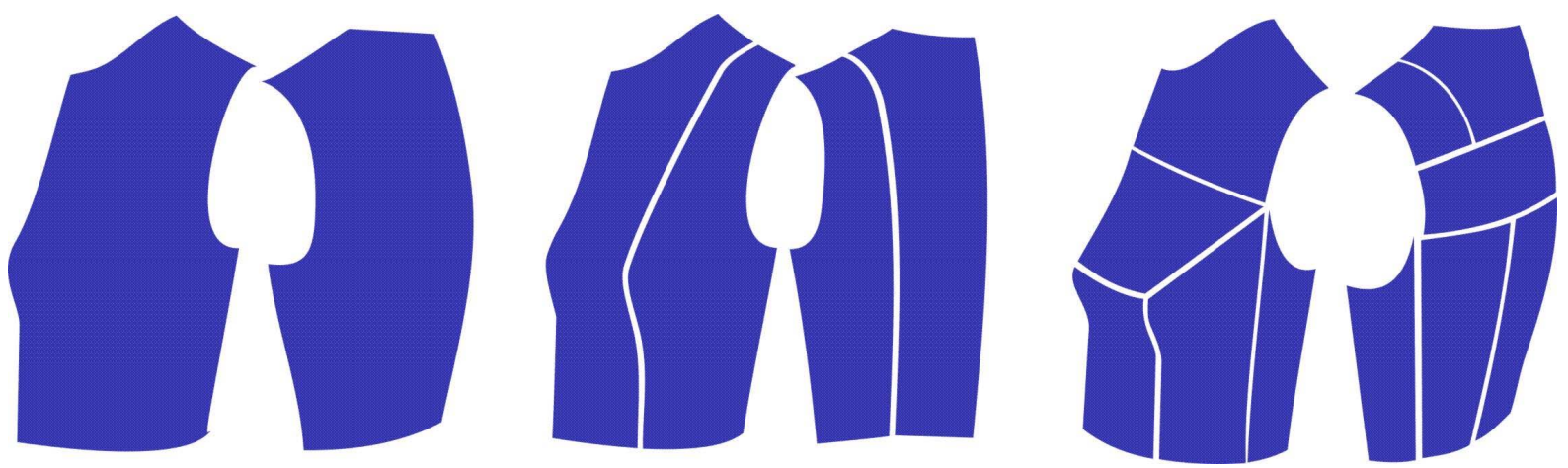

Fig.6. Three cases of dart division.

\section{Female shirt's templates from 3D to 2D}

\subsection{The data extraction of female shirt's model}

For female shirt point-cloud data extraction, the operation method and basic pattern data extraction is the same basically. Because the pattern is longer than the length of the female shirt, when the intercept point-cloud shows a grid as reference, the interception of hem need to be up to the length of the longest before interception. Then draw the hem curve horizontally, and intercept off curve within the point-cloud and get the hem of the arc (Fig. 7).

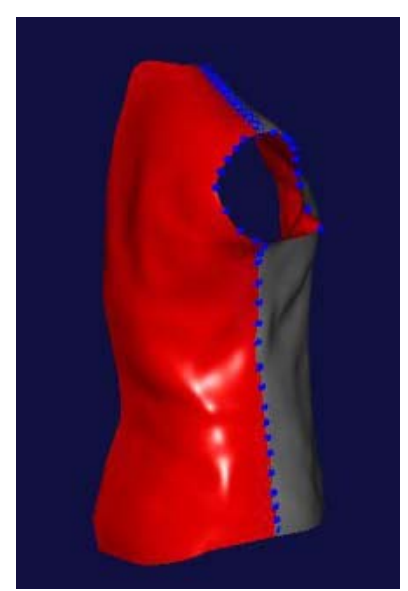

Fig. 7. The 3D modelling of experiment shirt.

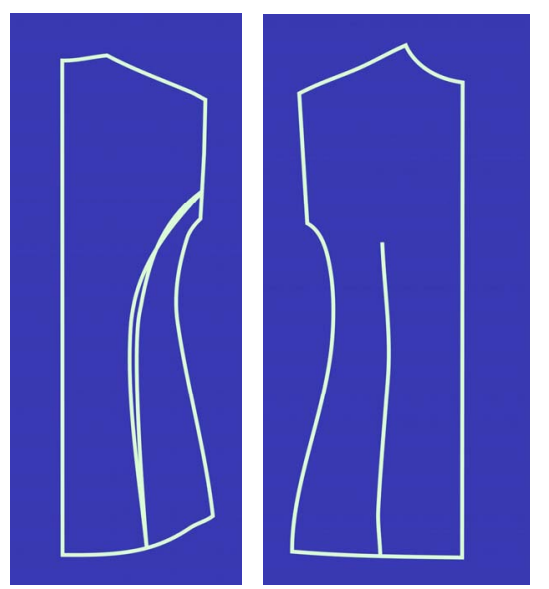

Fig. 8. The 2D templates of experiment shirt.

\subsection{Female shirt's templates from 3D to 2D}

In this experiment, using a based segmentation simple shirt style, darts were distributed based on basic pattern. Draw the line on the sheet of reconstruction surface front panel and back panel, and the process of operation and prototype surface is the same. The process of the divider need to adjust the shape to get the line position results of front panel and back panel.

In order to make the surface smooth and hold the shape of the center line and armhole, we need to extend the curve to the armhole and hem position to form the shape of the knife's back seam. In this way, it is very convenient to flat for back panel. We can obtain ideal shirt pattern after splicing methods such as modify the pattern a little bit.

The flatting process of shirt pattern is as same as basic pattern consistent. In order to ensure fabric smooth and flat, many to be done such as slightly modifying the back panel's length and the midline, former dart highway adjustment, dart extending to the armhole parts, dividing into two parts to unfold the pattern (Fig. 8). On the wireframe and surface DCTT system design platform, after flatting the shirt surface, measure the sample deformation process, firmness, perimeter and area for feasibility study and cost evaluation, and modify the pattern or shape to make finished products as close as possible to the product technical specifications ${ }^{[4]}$. 


\subsection{Testing}

For the pattern resulting from the basic pattern on both front panel and back panel, need to make the comparison of the corresponding parts' measurement. Comparing small shoulder width, neck line, front and back armhole, chest circumference, side seam, front panel and back panel front panel and back panel the center line, waist circumference in turn, the size of the parts is almost the same. Due to the surface with the triangular mesh stretching, there caused a tiny gap in different directions.

Production of female shirt sample fabric is grey fabric, final sample of front panel and back panel effect are shown in figure 8 with static mode of 160/84A. From wearing effect in this picture, the bust part of former is more fitting and the dart has basic straight shape. Side armhole has a good depth, but right shoulder blade can't attach. Because the fabric is proofed and shoulder part slightly prominent, the bottom arc curve is smooth. Overall, version type structure does not appear problems, so the wearing effect of front panel and back panel prove the rationality of the pattern (Fig. 9).
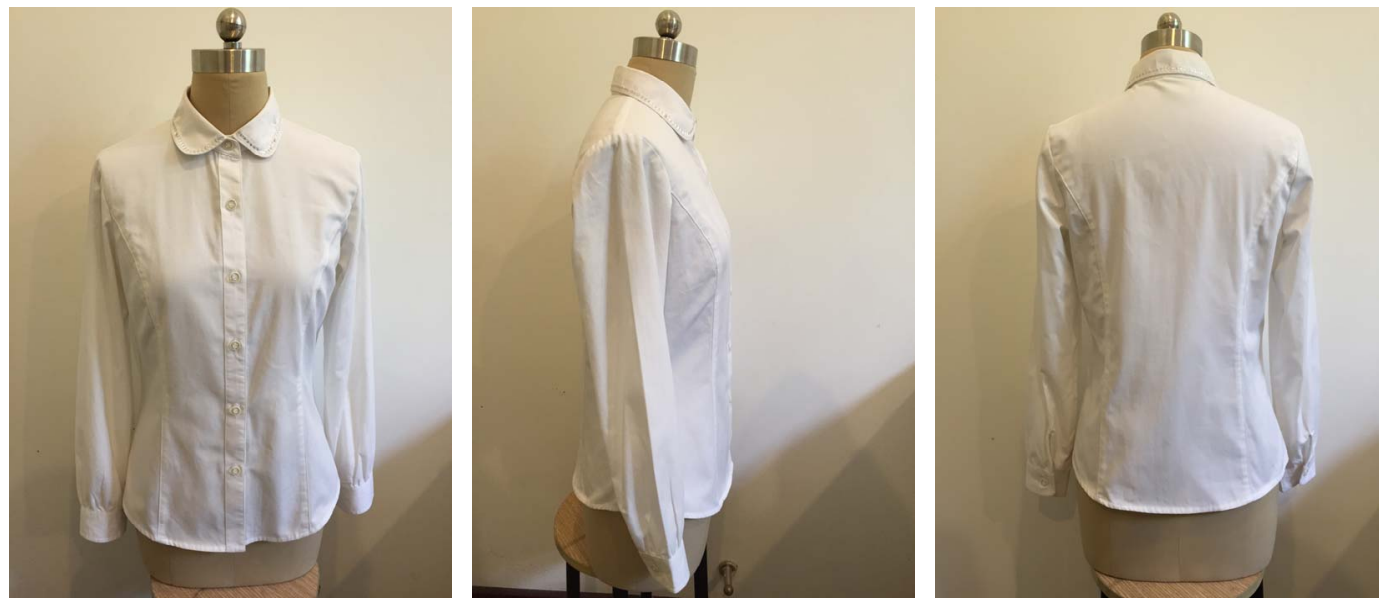

Fig. 9. Sample effect.

\section{Conclusions}

1) Data extracted with the method of non-contact body scanning, be able to be more scientific for handling the human body model.

2) Through comparing the plane plate method and the 3D model converted into $2 \mathrm{D}$ templates, it's be found that the latter is more docile for samples and can show better the relationship between the human body and clothing.

3) Can establish design database model to provide more convenient service for garment industrial production.

The key conversion technology's solve of clothing patterns from 3D modelling to $2 \mathrm{D}$ templates, that is the perfect combination of art and technology in this big-data era. This is also the clothing technology innovation, which means the trend of realizing high efficiency production and a new marketing mode of enterprises implement zero inventory marketing.

\section{References}

[1] Abu Sadat Muhammad Ssyen. Resilzablel outerwear tenplates for virtual design and pattern flattening [D]. Manchester: The University of Manchester, 2012:88-122.

[2] DENG Chuanshan, ZHANG Longlin lin, ZHOU Li. On establishing 3D model of standard human body for young men in southwest china[J]. China Textile Leader, 2015, 3: 68-69.

[3] SHANG Lige. The flattening of patterns based on Individual $s$ [D]. $\mathrm{Xi}$ 'an: $\mathrm{Xi}$ 'an Engineering University, 2015:29-35.

[4] WEI Huanyun, ZHOU Li. Virtual design of trousers pattern based on 3D scanning technology and related 2D templates [J]. China Textile Leader, 2014, 8: 64-65.

This research is supported by "Chongqing Social Science Fund (2014YBJJ027 \& 2014YBYS093)" and "Fundamental Research Funds for the Central Universities (XDJK2014A011)". 